\title{
Determinação do fator de proteção solar (FPS) in vitro e in vivo de emulsões com óleo de andiroba (Carapa guianensis)
}

\author{
Márcio Ferrari ${ }^{1 *}$, Maria S. C. Oliveira ${ }^{2}$,Adelino K. Nakano ${ }^{3}$,Pedro A. Rocha-Filho²
}

${ }^{\text {I} F a c u l d a d e ~ d e ~ F a r m a ́ c i a, ~ U n i v e r s i d a d e ~ d e ~ C u i a b a ́, ~ A v . ~ B e i r a ~ R i o ~ 3100, ~ J a r d i m ~ E u r o p a, ~ 78015-480, ~ C u i a b a ́, ~ M T, ~}$ Brasil,

${ }^{2}$ Faculdade de Ciências Farmacêuticas de Ribeirão Preto, Universidade de São Paulo, Avenida Zeferino Vaz s/n, Monte Alegre, 14040-903, Ribeirão Preto, SP, Brasil,

${ }^{3}$ Evic Brasil, Avenida Indianópolis 1455, Planalto Paulista, 04063-002, São Paulo, SP, Brasil

\begin{abstract}
RESUMO: O óleo de andiroba extraído da Carapa guianensis tem apresentado considerável interesse pelas indústrias farmacêutica e cosmética devido a suas propriedades antiinflamatória e repelente de insetos. Esta pesquisa teve como objetivo avaliar a atividade fotoprotetora in vitro e in vivo do óleo de andiroba. Emulsões estáveis do tipo $\mathrm{O} / \mathrm{A}$ foram preparadas com o metoxicinamato de etilhexila aditivadas ou não com óleo de andiroba. As características organolépticas foram avaliadas através de análises macroscópicas e o tipo de emulsão pelo teste de diluição. O FPS in vitro foi determinado pelo método espectrofotométrico desenvolvido por Mansur, e o teste in vivo de acordo com a metodologia padronizada pela Food and Drug Administration (FDA). Não houve diferença estatística entre as formulações, demonstrando que o óleo de andiroba não apresentou atividade fotoprotetora.
\end{abstract}

Unitermos: Carapa guianensis, Meliaceae, fator de proteção solar (FPS), óleo de andiroba.

\begin{abstract}
In vitro and in vivo determinations of sun protection factor (SPF) of emulsions with andiroba oil (Carapa guianensis)". The andiroba oil extracted of Carapa guianensis has attracted considerable interest by the pharmaceutical and cosmetic industries because of its anti-inflammatory and insect repellent properties. This research evaluated the in vitro and in vivo andiroba oil photoprotection activity. Stable $\mathrm{O} / \mathrm{W}$ emulsions with ethylhexyl methoxycinnamate were prepared with and without andiroba oil. These emulsions were assessed by evaluating several parameters such as macroscopic analysis and organoleptic aspects, emulsion type by diluition test and in vitro and in vivo determinations of Sun Protect Factor (SPF). The in vitro SPF was determined according to the spectrophotometric method developed by Mansur. The in vivo SPF values were determined according to the Food and Drug Administration (FDA) method. There was not statistical difference among the formulations, demonstrating that the andiroba oil did not present photoprotective activity.
\end{abstract}

Keywords: Carapa guianensis, Meliaceae, sun protection factor (SPF), andiroba oil.

\section{INTRODUÇÃO}

No Brasil, o número de casos de câncer de pele tem aumentado, representando um considerável problema de saúde pública (INCA, 2006). Portanto, diversos produtos têm sido desenvolvidos aditivados com filtros solares.

Diante de tantos produtos contendo filtros solares disponíveis no mercado, industrializados ou manipulados, a dúvida no momento da aquisição é crucial. De acordo com os resultados de pesquisas, um terço da população escolhe essa categoria de produtos pelas informações contidas na embalagem (Johnson; Lookingbill, 1984). Tal afirmação leva a uma preocupação ainda maior em oferecer informações, precisas e confiáveis, principalmente no que diz respeito à proteção oferecida pelo produto. No entanto, é relevante destacar que a segurança e eficácia dos produtos que contém filtros solares não estão centrados apenas no desenvolvimento do produto, mas também na utilização 
correta por parte do consumidor (Lademann et al., 2004; Szepietowski et al., 2004; Stengel; Fernandez, 2005).

Uma das tendências do mercado cosmético é o desenvolvimento de produtos com o maior número de componentes de origem natural, especialmente os de origem vegetal, explorando de forma racional a biodiversidade brasileira. A incorporação de ativos naturais em produtos cosméticos tem sido uma prática corrente, pois há grande interesse tanto do mercado nacional quanto do internacional pelos mesmos, principalmente se a matéria-prima apresenta estudos científicos comprovando a segurança e eficácia além do comprometimento com o desenvolvimento sustentável (Franquilino, 2006a). Cabe destacar também que o consumidor está cada vez mais crítico e exigente optando pelo uso de produtos cosméticos que sejam de origem natural, com qualidade cientificamente comprovada.

O gênero Carapa é composto por duas espécies, C. procera e C. guianensis. A primeira espécie ocorre na África e América do Sul (Guiana Francesa, Suriname e Brasil), enquanto que a segunda, $C$. guyanensis ocorre da América Central até o norte da América do Sul (Venezuela, Equador, Colômbia, Peru e Brasil). No Brasil as duas espécies ocorrem principalmente no estado do Amazonas e são conhecidas como andiroba, andirova, carapinha e iandiroba (Fisch et al., 1995; Ferraz; Sampaio, 1996; Vinson et al., 2005; Agra et al., 2007).

O óleo extraído desta árvore tem apresentado grande interesse pelas indústrias farmacêutica $\mathrm{e}$ cosmética. Dentre suas propriedades destacam-se a ação antiinflamatória (Ferrari, 1998; Penido et al., 2006a) de repelente de inseto (Miot et al., 2004; Mendonça et al., 2005) e mais recentemente foi descrito as ações anti alérgica e analgésica (Penido et al., 2006b,c). Também tem sido utilizado popularmente em algumas regiões do país, pelos "raizeiros" e vendedores de mercados populares com o apelo de "ser bom para evitar câncer de pele, por proteger dos danos do sol", apresentando assim uma suposta atividade fotoprotetora.

Fundamentados nos conhecimentos populares o presente trabalho teve como objetivo avaliar a eficácia fotoprotetora in vitro e in vivo do óleo de andiroba em emulsões óleo em água (O/A).

\section{MATERIAL E MÉTODOS}

Como fase oleosa foi utilizado o óleo de andiroba - Carapa guaianensis óleo da semente (Beraca Ingredients). Como tensoativo foi usado o trilaureth4-fosfato (Hostaphat ${ }^{\circledR}$ KL 340 NB - Clariant S.A.). A fase aquosa foi constituída de água recém destilada e poliacrilamida, $\mathrm{C}_{13-14}$ isoparafina, laureth-7 (Sepigel $^{\circledR}$ 305 - Chemyunion Química Ltda). Como filtro solar o metoxicinamato de etilhexila (Escalol 557 - ISP do Brasil Ltda); e os seguintes coadjuvantes técnicos: Nipagin (metilparaben), nipasol (propilparaben) e propileno glicol, BHT, palmitato de ascorbila, estearato de glicerila e ácido cítrico (Oxynex ${ }^{\circledR} 2004$ - Merck S.A.). As denominações das matérias-primas foram apresentadas de acordo com a International Nomenclature Cosmetics Ingredients (INCI) (ICID, 2000).

\section{Emulsão primária}

Utilizou-se a emulsão primária preparada pelo método de inversão de fases a frio padronizada e estabilizada por Ferrari (2002).

\section{Análise macroscópica}

Após vinte e quatro horas do preparo das amostras foram observadas as características organolépticas e a homogeneidade das formulações.

\section{Determinação do tipo de emulsão}

Realizado com o objetivo de identificar o tipo de emulsão através do teste de diluição (Prista et al., 1996).

\section{Determinação in vitro do Fator de Proteção Solar (FPS)}

Foi determinado pelo método espectrofotométrico (Espectrofotômetro Spectronic ${ }^{\circledR}$ Genesys $^{\mathrm{TM}}$ 5) descrito por Mansur (1986a). Os resultados foram calculados pelos valores originais $(n=9)$ e expressos pela média. Foram analisados estatisticamente pelo teste t-Student com correção de Welch's e pelo teste ANOVA. Valores com $\mathrm{p}<0,05$ foram considerados estatisticamente significativos.

\section{Determinação in vivo do Fator de Proteção Solar (FPS)}

Realizado pela Evic Brasil, de acordo com o método da Food and Drug Administration (FDA) (1999). O Protocolo de Pesquisa e Consentimento Livre e Esclarecidos foram aprovados pelo Comitê de Ética em Pesquisa da Faculdade de Medicina do ABC, sob o protocolo $n^{\circ} 99 / 2001$. Os resultados foram calculados pelos valores originais $(n=10)$ e expressos pela média. Os dados foram submetidos ao mesmo tratamento estatístico realizado no teste de determinação in vitro do FPS.

\section{RESULTADOS E DISCUSSÃO}

Com o objetivo de verificar a ação fotoprotetora do óleo de andiroba foram estudadas duas formulações (Tabela 1) padronizadas e estabilizadas por Ferrari (2002).

A emulsão contendo óleo de andiroba (F1) 
Tabela 1. Composição das formulações estudadas.

\begin{tabular}{lcc}
\hline \multicolumn{1}{c}{ Composição } & $\begin{array}{c}\text { Fórmula 1 (F1) } \\
(\% \mathrm{p} / \mathrm{p})\end{array}$ & $\begin{array}{c}\text { Fórmula 2 (F2) } \\
(\% \mathrm{p} / \mathrm{p})\end{array}$ \\
\hline $\begin{array}{l}\text { Carapa guaianensis, óleo das sementes } \\
\text { Trilaureth-4-phosphate }\end{array}$ & 11,35 & --- \\
Propilparaben & 1,00 & 1,00 \\
Propileno glicol, BHT, palmiato de ascorbila, & 0,05 & 0,05 \\
estearato de glicerila e ácido cítric & & \\
Metoxicinamato de etilhexila & 0,10 & 0,10 \\
Poliacrilamida, C 13-14 isoparafina e Laureth-7 & 3,75 & 3,75 \\
Metilparaben & 2,00 & 2,00 \\
Água destilada & 0,15 & 0,15 \\
\hline
\end{tabular}

Tabela 2. Determinação do valor do Fator de Proteção Solar (FPS) in vitro e in vivo das formulações em estudo.

\begin{tabular}{lcc}
\hline \multicolumn{1}{c}{ Composição } & FPS \\
\hline & in vitro & in vivo \\
Emulsão primária com óleo de andiroba (F1) & $6,64 \pm 0,15$ & $6,24 \pm 1,56$ \\
Emulsão primária sem óleo de andiroba (F2) & $6,54 \pm 0,16$ & $5,72 \pm 1,13$ \\
Emulsão com 8\% de Homossalato (Padrão) & $4,12 \pm 0,16$ & $4,00 \pm 0,30$ \\
\hline
\end{tabular}

Os valores foram calculados pelos valores originais (in vitro com $n=9$ e in vivo com $n=10$ ) e expressos pela média \pm desvio padrão.

(Tabela 1) apresentou-se macroscopicamente com aspecto de creme, cor bege e odor característico do óleo, e a fórmula 02 (Tabela 1), caracterizou-se pela cor branca com o mesmo aspecto.

Pelo teste de diluição verificou-se que as formulações apresentaram-se do tipo O/A. Estes resultados reforçam a indicação da natureza hidrofílica do tensoativo. Skrypzak et al. (1980) descreveram que o éster fosfórico utilizado (Hostaphat ${ }^{\circledR}$ KL 340 NB), é um tensoativo aniônico, com elevado caráter hidrofílico e favorece a formação de emulsões estáveis por promover a formação de rede cristalina na interface O/A.

Seguindo a tendência da utilização de produtos naturais na produção de cosméticos, várias pesquisas têm sido desenvolvidas com o objetivo de verificar a ação fotoprotetora de extratos e óleos vegetais que contêm, em sua constituição, flavonóides, taninos, antraquinonas, alcalóides e os polifenóis (Bobin et al., 1994; Tabrizi et al., 2003; Souza et al., 2005; Di Mambro; Fonseca 2006).

Ao analisar a fitoquímica do óleo de andiroba (Correa, 1984; Qi et al., 2004) verificou-se que não há metabólitos com possível ação fotoprotetora, no entanto justifica-se a realização deste trabalho, baseado nas evidências populares e também na possibilidade da interação dos componentes da formulação que podem propiciar aumento do FPS (Ramos et al., 1996; Hu; Wang, 1998; Tabrizi et al., 2003; Souza et al., 2005).

Os resultados das determinações dos valores do FPS in vitro e in vivo estão demonstrados na Tabela 2.

A emulsão com $8 \%$ de homossalato foi utilizada como padrão e para controle da técnica, seguindo as especificações descritas na metodologia in vivo para determinação do FPS padronizada pela FDA (FDA, 1999).

As emulsões primárias do tipo $\mathrm{O} / \mathrm{A}$, contendo $3,75 \%(\mathrm{p} / \mathrm{p})$ de metoxicinamato de etilhexila aditivadas ou não de óleo de andiroba, apresentaram pelos testes in vitro e in vivo FPS 6, o que demonstra uma boa correlação entre os resultados das metodologias utilizadas (Mansur et al., 1986b; Santos et al., 1999).

O tratamento estatístico pelo teste $\mathrm{t}$ - Student com correção de Welch's $(\mathrm{p}<0,05)$ foi utilizado para avaliar as formulações aos pares, in vitro e in vivo, contendo ou não óleo de andiroba. Pela análise estatística, o FPS não apresentou diferença significante entre as formulações estudadas. Através do teste ANOVA, que avaliou todas as formulações em único tratamento, também não apresentaram diferenças significantes. Portanto, o óleo em estudo, não apresentou ação fotoprotetora, confrontando desta forma com um dos seus usos populares.

\section{CONCLUSÃO}

Apesar do óleo de andiroba não apresentar ação fotoprotetora, não deve ser desconsideradas suas atividades emoliente (Franquilino, 2006b), antiinflamatória (Ferrari, 1998; Penido et al., 2006a) e de repelente de insetos (Miot et al., 2004; Mendonça et al., 2005) que podem ser benéficas para o consumidor em formulações fotoprotetoras e até mesmo servir como um apelo de marketing para o produto. 


\section{AGRADECIMENTOS}

Os autores agradecem o apoio da FCFRP-USP, da UNIC e em especial à EVIC BRASIL pela gentileza da realização dos testes in vivo de determinação do Fator de Proteção Solar (FPS). Agradecem também as empresas: Beraca, Chemyunion, Clariant e ISP pela doação das matérias-primas.

\section{REFERÊNCIAS}

Agra MF, França PF, Barbosa-Filho JM 2007. Synopsis of the plants known as medicinal and poisonous in Northeast of Brazil. Rev Bras Farmacogn 17: 114-140.

Bobin MF, Raymond M, Martini MC 1994. UVA/UVB absorption properties of natural products. Cosmet Toiletries 109: 63-78.

Correa P 1984. Dicionário de planta úteis do Brasil e exóticas cultivadas. Brasília: IBDF, p. 113-114, 371-376.

Di Mambro VM, Fonseca MJV 2006. Avaliação da eficácia fotoprotetora in vivo de formulações contendo extratos de Ginkgo biloba e Glycirrhiza glabra. XX Congresso Brasileiro de Cosmetologia. São Paulo, Brasil.

FDA 1999. Federal Register. Food and Drug Administration. v. 64, n. 98.

Ferrari M 2002. Desenvolvimento e avaliação da eficácia fotoprotetora de emulsões múltiplas contendo metoxicinamato de etilhexila e óleo de andiroba (Carapa guyanensis). Ribeirão Preto, 142f. Tese de Doutorado - Faculdade de Ciências Farmacêuticas de Ribeirão Preto, Universidade de São Paulo.

Ferrari M 1998. Obtenção e aplicação de emulsões múltiplas contendo óleos de andiroba e copaíba. Ribeirão Preto, 147f. Dissertação de Mestrado Faculdade de Ciências Farmacêuticas de Ribeirão Preto, Universidade de São Paulo.

Ferraz IDK, Sampaio PTB 1996. Métodos simples de armazenamento das sementes de andiroba (Capara guianensis Aubl. e Carapa procera D.C.- Meliaceae). Acta Amazonica 26: 137-144.

Fisch STV, Ferraz IDK, Rodrigues WA 1995. Distinguishing Carapa guianensis Aubl. from Carapa procera D.C. (Meliaceae) by morphology of young seedlings. Acta Amazonica 25: 193-200.

Franquilino E 2006a. Em ritmo de expansão. Cosmet Toiletries (ed. Port.), edição temática, 18: 7-10.

Franquilino E 2006b. Ativos Amazônicos. Cosmet Toiletries (ed. Port.), edição temática, 18: 18-53.

Hu G, Wang X 1998. Research on a natural sunscreen from Chinese herbs. Int J Cosmet Sci 20: 175-181.

ICID 2000. International cosmetic ingredient dictionary and handbook 2000. Washington: CTFA, 3v.

INCA 2006. http://www.inca.gov.br, acessada em junho de 2006.

Johnson EY, Lookingbill DP 1984. Sunscreen use and sun exposure, trends in a white population. Arch Dermatol 120: 727-731.

Lademann J, Schanzer S, Richter H, Pelchrzim RV, Zastrow L, Golz K, Sterry W 2004. Sunscreen application at the beach. J Cosmet Dermatol 3: 62-68.

Mansur JS, Breder MNR, Mansur MCA, Azulay RD 1986a.
Determinação do fator de proteção solar por espectrofotometria. An Bras Dermatol 61: 121-124.

Mansur JS, Breder MNR, Mansur MCA, Azulay RD 1986b. Correlação entre a determinação do fator de proteção solar em seres humanos e por espectrofotometria. $A n$ Bras Dermatol 61: 167-172.

Mendonça FAC, Silva KFS, Santos KK, Ribeiro-Júnior KAL, Sant'Ana AEG 2005. Activities of some Brazilian plants against larvae of the mosquito Aedes aegypti. Fitoterapia 76: 629-636.

Miot HA, Batistella RF, Batista KA, Volpato DEC, Augusto LST, Madeira NG, Haddad-Júnior V, Miot LDB 2004. Comparative study of the topical effectiveness of the andiroba oil (Carapa guianensis) and DEET $50 \%$ as repellent for Aedes sp. Rev Inst Med Trop Sao Paulo 46: 253-256.

Penido C, Conte FP, Chagas MSS, Rodrigues CAB, Pereira JFG, Henriques MGMO 2006a. Antiinflammatory effects of natural tetranortriterpenoids isolated from Carapa guianensis Aublet on zymosan-induced arthritis in mice. Inflamm Res 55: 457-464.

Penido C, Costa KA, Pennaforte RJ, Costa MFS, Pereira JFG, Siani AC, Henriques MGMO 2006b. Anti-allergic effects of natural tetranortriterpenoids isolated from Carapa guianensis Aublet on allergen-induced vascular permeability and hyperalgesia. Inflamm Res 54: 295-303.

Penido C, Costa KA, Costa MF, Pereira JF, Siani AC, Henriques MG 2006c. Inhibition of allergen-induced eosinophil recruitment by natural tetranortriterpenoids is mediated by the suppression of IL-5, CCL11/eotaxin and NFkappaB activation. Int Immunopharmacol 6: 109-121.

Prista LN, Alves AC, Morgado R 1996. Tecnologia Farmacêutica. Lisboa: Fundação Calouste Gulbenkian. v. 1.

Qi SH, Wu DG, Zhang S, Luo XD 2004. Constituents of Carapa guianensis Aubl. (Meliaceae). Pharmazie 59: 488-490.

Ramos MFS, Stantos EP, Blzarri CHB, Mattos HA 1996. Preliminary studies towards utilization of various plant extracts as antisolar agents. Int J Cosmet Sci 18: 87-101.

Santos EP, Freitas ZM, Souza KR, Garcia S, Vergnanini A. 1999. In vitro and in vivo determinations of sun protection factors of sunscreen lotions with octylmethoxycinnamate. Int J Cosmet Sci 21: 1-5.

Skrypzak W, Reng A, Quack JM 1980. Formulating cosmetic emulsions with o-phosphoric ester. Cosmet Toileties 95: 47-56.

Souza TM, Santos LE, Moreira RRD, Isaac VLB 2005. Avaliação da atividade fotoprotetora de Achillea millefolium L. Rev Bras Farmacogn 15: 36-38.

Stengel FM, Fernandez JF 2005. Education and behavioral change for sun protection. $J$ Cosmet Dermatol 4: 83-88.

Szepietowski JC, Nowicka D, Reich A, Melon M 2004. Application of sunscreen preparations amog young Polish people. J Cosmet Dermatol 3: 69-72.

Tabrizi H, Mortazavi AS, Kamalinejad M 2003. An in vitro evaluation of various Rosa damascena flower extracts as a natural antisolar agent. Int J Cosmet Sci 25: 259-265. 
Vinson CC, Azevedo VCR, Sampaio I, Ciampi AY 2005. Development of microsatellite markers for Carapa guianensis (Aublet), a tree especies from Amazon forest. Mol Ecol Notes 5: 33-34. 